\title{
Structure-Activity Relationships of Constrained Dermorphin Analogues Containing an $\alpha$-Alkyl- $\beta$-Substituted Alanines
}

\author{
Anika Lasota ${ }^{1}$, Oliwia Frączak ${ }^{1}$, Adriana Muchowska ${ }^{2}$, Aleksandra Misicka ${ }^{2}$, \\ Michał Nowakowski ${ }^{3}$, Maciej Maciejczyk ${ }^{4}$, Andrzej Ejchart ${ }^{5}$, \\ and Aleksandra Olma ${ }^{1}$ \\ ${ }^{1}$ Institute of Organic Chemistry, Lodz University of Technology, Zeromskiego, 116,90-924, Lodz; ${ }^{2}$ Mossakowski \\ Medical Research Centre, Polish Academy of Sciences, Pawinskiego 5, 01-793, Warsaw; ${ }^{3}$ Centre of New \\ Technologies, University of Warsaw, Zwirki $i$ Wigury 93, 02-089, Warsaw; ${ }^{4}$ Chair of Physics and Biophysics, \\ University of Warmia and Mazury ul. Oczapowskiego 4, 10-719, Olsztyn; ${ }^{5}$ Institute of Biochemistry and \\ Biophysics, Polish Academy of Sciences, Pawinskiego 5A, 02-106, Warsaw, Poland
}

\section{Introduction}

Narcotic analgesics produce pain relief through the activation of $\mu$-opioid receptors (MOR), but the use of these drugs is limited by their side effects. Endogenous opioid peptides have been studied since their discovery with a view to developing effective analgesics. Dermorphin, an opioid heptapeptide (Tyr- $(R)$-Ala-Phe-Gly-Tyr-Pro-Ser- $\mathrm{NH}_{2}$ ) isolated from the skin of a South American frog, shows remarkably high $\mu$ selectivity and an extremely potent antinociceptive effect [1]. To understand the interactions between MOR ligands and the receptor, more than 100 dermorphin analogues have been synthesized and their structure-activity relationships have been investigated. The use of peptides as drugs is restricted because of their rapid biodegradation and low selectivity connected to high conformational freedom. These disadvantageous properties can be remedied by the synthesis of peptidomimetics, which are biologically active compounds with high selectivity and long half-lives. Non-coded amino acids offer a convenient tool for the modification of bioactive peptides and for conformational studies as they can be used to induce certain conformations. In these applications, of particular importance are $\alpha, \alpha$-disubstituted glycines. This work reports the synthesis and receptor binding of dermorphin analogues containing $(R)$ or $(S)$ - $\alpha$-alkyl- $\beta$-(azido, 1-piperidinyl)alanines at position 2 or 3 (Figure 1).

\section{Results and Discussion}

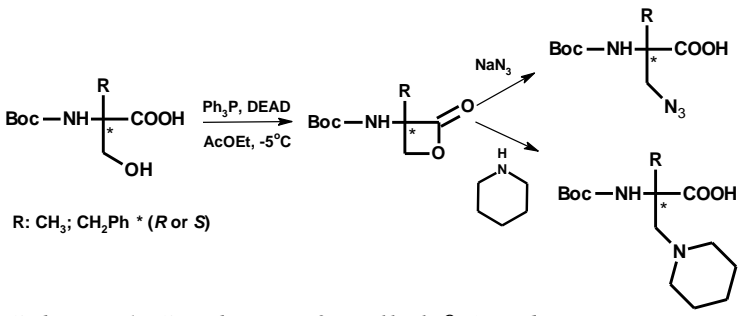

Scheme 1. Synthesis of $\alpha$-alkyl- $\beta$-(azido, 1-piperidinyl) alanines.
An efficient method of synthesizing multifunctional $\alpha, \alpha$-disubstituted glycines from easily accessible $\alpha$-alkylserines has been developed by our research team [2]. Racemic $N$-Boc- $\alpha$-alkylserine has been resolved by fractional crystallization of diastereoisomeric salts with (-)-ephedrine. The cyclization of $(R)$ or $(S)$-Boc- $\alpha$-alkylserines under Mitsunobu conditions leads to the corresponding $\beta$-lactones in high yields. The ring opening of $N$-protected $(R)$ or $(S) \alpha$-alkylserine $\beta$-lactones
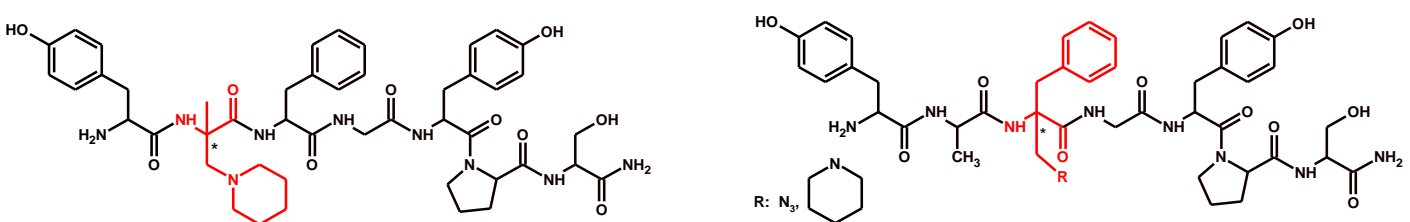

Fig. 1. Primary structures of synthesized dermorphin analogues modified at position 2 or 3 with $(R)$ or (S)- $\alpha$-alkyl- $\beta$-(1-piperidinyl)alanines. 
with sodium azide or piperidine as a nucleophile gives a variety of unnatural $\alpha, \beta$-disubstitiuted amino acids (Scheme 1).

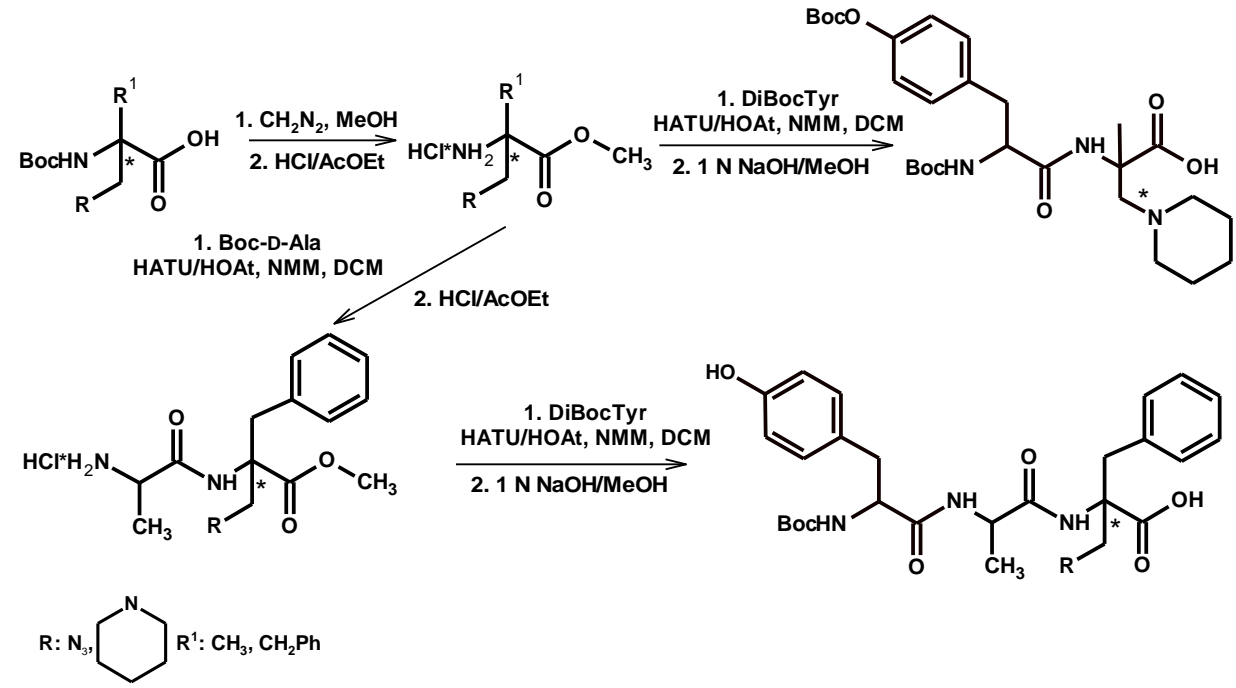

Scheme 2. Synthesis of di-and tripeptides containing (R) or (S) $\alpha$-alkyl- $\beta$-azido(1-piperidinyl)alanine.

Our preliminary experiments showed that the classic (step-by-step) synthesis of deltorphin I containing $\alpha$-methyl- $\beta$-azidoalanine on solid phase using the Boc strategy was not effective. The inability to control a critical stage (the acylation of the $\alpha, \alpha$-disubstituted amino group in the amino acid) forced us to change the strategy of synthesis. We decided to synthesize dipeptides and tripeptides containing $\alpha, \alpha$-disubstituted amino acids in solution (Scheme 2), using reagents for difficult couplings (HATU/HOAt). The $\mathrm{N}, \mathrm{O}$-protected di(tri)peptides were used for the acylation of penta(tetra)peptides (Gly-Tyr-Pro-Ser-NH $\mathrm{NH}_{2}$, Phe-Gly-Tyr-Pro-Ser- $\mathrm{NH}_{2}$ ) in manual solid-phase peptide synthesis (SPPS). The crude peptides were purified by RP-HPLC. The purity of the final TFA salts was assessed by analytical HPLC (purity 97\%-99\%) and ESI-MS. The new analogues of dermorphin were tested for selectivity and affinity to $\delta$ - and $\mu$-opioid receptors in a standard displacement test on receptors in rat brain homogenates (Table 1). It was found that the introduction of $(R$ or $S)$ - $\alpha$-methyl- $\beta-(1$ piperidinyl)alanines at position 2 in DERM causes a significant decrease of activity. The effect of substituting $\mathrm{Phe}^{3}$ with $\alpha$-benzyl- $\beta$-azido(or 1-piperidinyl)alanines in dermorphin depends on the chirality of the amino acid. The incorporation of $(R)$ enantiomers results in analogues with higher selectivity and affinity to opioid receptors (I and III), whereas analogues with the $(S)$ configuration are less potent (II and IV). This can be explained by analogy between $(R)$ - $\alpha$-benzyl- $\beta$-azido(or 1 piperidinyl)alanines and $(S)$ phenylalanine, which have the same steric orientation of the aromatic ring. The incorporation of $(R)$ - $\alpha$-benzyl- $\beta$-azidoalanine at position 3 in dermorphin gives an active analogue with $\mu$ selectivity, but with moderate affinity to the $\delta$-receptor.

NMR experiments were conducted with the two most biologically active analogues modified with $\alpha$-benzyl- $\beta$ - $(R$ and $S)$-azidoalanines (I and II). The nuclear Overhauser effect (NOE) has become the method of choice in studying conformations of organic and biological molecules. Short linear peptides are usually characterized by high structural flexibility. Therefore, long range correlations in their NOESY/ROESY spectra have been seldom observed. Nevertheless, one could expect increased conformational rigidity in peptides containing $\alpha, \alpha$-disubstituted amino acid residues. In order to explain this issue, an NMR study was carried out for the dermorphin analogue containing $(R)$ - $\alpha$-benzyl$\beta$-azidoalanine (I) and its diastereoisomer modified with $(S)$ - $\alpha$-benzyl- $\beta$-azidoalanine (II). An initial analysis of the spectrum revealed two distinct sets of signals due to cis:trans isomerization of the $\mathrm{Tyr}^{5}$ Pro $^{6}$ amide bond in 1:4 ratio. The distinction between cis:trans isomers was based on $\mathrm{C} \gamma$ and $\mathrm{C} \delta$ chemical shifts and the relative intensity of the signals (Table 2). 
Table 1. Binding affinities of new dermorphin analogues to $\delta$ - and $\mu$-opioid receptors.

\begin{tabular}{|c|c|c|c|}
\hline \multirow{2}{*}{ PEPTIDE } & \multicolumn{3}{|c|}{$I C_{50}(n M)$} \\
\hline & $\mu^{a}$ & $\delta^{b}$ & Select. \\
\hline Tyr-(R)-Ala-Phe-Gly-Tyr-Pro-Ser-NH 2 (dermorphin) [3] & 1.22 & $178.6^{\mathrm{c}}$ & 146 \\
\hline $\operatorname{Tyr}-(R)$-Ala- $(R)$ - $\alpha$-benzyl- $\beta$-azidoAla-Gly-Tyr-Pro-Ser- $\mathrm{NH}_{2}[4](\mathbf{I})$ & 4.29 & 22.6 & 5.27 \\
\hline Tyr- $(R)$-Ala- $(S)$ - $\alpha$-benzyl- $\beta$-azidoAla-Gly-Tyr-Pro-Ser-NH $2[4]$ (II) & 151 & 1057 & 7 \\
\hline Tyr- $(R)$-Ala- $(R)$ - $\alpha$-benzyl- $\beta$-(1-piperidinyl)Ala-Gly-Tyr-Pro-Ser-NH 2 (III) & 52.48 & $>10000$ & $>191$ \\
\hline Tyr- $(R)$-Ala- $(S)$ - $\alpha$-benzyl- $\beta-\left(1-\right.$ piperidinyl)Ala-Gly-Tyr-Pro-Ser- $\mathrm{NH}_{2}(\mathbf{I V})$ & 208.9 & $>10000$ & $>48$ \\
\hline Tyr- $(R)-\alpha$-methyl- $\beta$-(1- piperidinyl)Ala-Phe-Gly-Tyr-Pro-Ser- $\mathrm{NH}_{2}(\mathbf{V})$ & 204.2 & $>10000$ & $>52$ \\
\hline Tyr-(S)- $\alpha$-methyl- $\beta-\left(1-\right.$ piperidinyl)Ala-Phe-Gly-Tyr-Pro-Ser- $\mathrm{NH}_{2}(\mathbf{V I})$ & $>10000$ & $>10000$ & - \\
\hline
\end{tabular}

${ }^{a}$ versus $\left[{ }^{3} H\right] D A M G O,{ }^{b}$ versus $\left[{ }^{3} H\right] D E L T,{ }^{c}$ versus DADLE.

Table 2. ${ }^{1} \mathrm{H}$ and ${ }^{13} \mathrm{C}$ chemical shifts (in ppm) of Tyr-(R)-Ala-(S and $\left.R\right)-\alpha$-benzyl- $\beta$-azidoAla-Phe-AspVal-Val-Gly- $\mathrm{NH}_{2}$ (Aaa= $\alpha$-benzyl- $\beta$-azidoalanine).

\begin{tabular}{|c|c|c|c|c|c|c|c|c|c|c|c|c|c|}
\hline AA & $\mathrm{HN}$ & $\mathrm{H} \alpha$ & $\mathrm{H} \beta$ & $\mathrm{H} \gamma$ & $\mathrm{H} \delta$ & $\mathrm{H} \varepsilon$ & $\mathrm{H} \zeta$ & $\mathrm{Ca}$ & $\mathrm{C} \beta$ & $\mathrm{C} \gamma$ & $\mathrm{C} \delta$ & $\mathrm{C} \varepsilon$ & $\mathrm{C} \zeta$ \\
\hline $\begin{array}{r}\operatorname{Tyr}^{1} S \\
R\end{array}$ & $\begin{array}{l}\text { n.a. } \\
\text { n.a. }\end{array}$ & $\begin{array}{l}4.087 \\
4.090\end{array}$ & $\begin{array}{l}3.057 \\
3.120 \\
3.071 \\
3.123\end{array}$ & $\begin{array}{l}-- \\
---\end{array}$ & $\begin{array}{l}7.112 \\
7.108\end{array}$ & $\begin{array}{l}6.869 \\
6.867\end{array}$ & $\begin{array}{l}-- \\
---\end{array}$ & $\begin{array}{l}\text { n.a. } \\
57.54\end{array}$ & $\begin{array}{l}\text { n.a. } \\
38.91\end{array}$ & $\begin{array}{l}\text { n.a. } \\
\text { n.a. }\end{array}$ & $\begin{array}{l}133.44 \\
133.43\end{array}$ & $\begin{array}{l}118.65 \\
118.62\end{array}$ & $\begin{array}{l}\text { n.a. } \\
\text { n.a. }\end{array}$ \\
\hline $\begin{array}{r}\mathrm{Ala}^{2} S \\
R\end{array}$ & $\begin{array}{l}8.413 \\
8.364\end{array}$ & $\begin{array}{l}4.251 \\
4.277\end{array}$ & $\begin{array}{l}1.161 \\
1.176\end{array}$ & --- & $\begin{array}{l}--- \\
---\end{array}$ & --- & $\begin{array}{l}--- \\
---\end{array}$ & $\begin{array}{l}52.36 \\
52.33\end{array}$ & $\begin{array}{l}19.09 \\
\text { n.a. }\end{array}$ & --- & --- & --- & --- \\
\hline $\mathrm{Aaa}^{3} S$ & $\begin{array}{l}8.825 \\
8.360\end{array}$ & $\begin{array}{l}\text { benzyl } \\
\mathrm{CH}_{2} \mathrm{~N}_{3} \\
\text { benzyl } \\
\mathrm{CH}_{2} \mathrm{~N}_{3}\end{array}$ & $\begin{array}{l}3.093 \\
3.262 \\
3.613 \\
3.659 \\
3.112 \\
3.202 \\
3.647 \\
3.768\end{array}$ & $\begin{array}{l}--- \\
--- \\
--- \\
---\end{array}$ & $\begin{array}{l}7.087 \\
--- \\
7.085 \\
---\end{array}$ & $\begin{array}{l}7.322 \\
--- \\
7.313 \\
---\end{array}$ & $\begin{array}{l}7.321 \\
--- \\
7.313 \\
---\end{array}$ & $\begin{array}{l}\text { benzyl } \\
\mathrm{CH}_{2} \mathrm{~N}_{3} \\
\text { benzyl } \\
\mathrm{CH}_{2} \mathrm{~N}_{3}\end{array}$ & $\begin{array}{l}40.72 \\
54.93 \\
40.71 \\
54.37\end{array}$ & $\begin{array}{l}\text { n.a. } \\
--- \\
\text { n.a. } \\
---\end{array}$ & $\begin{array}{l}132.98 \\
--- \\
133.01 \\
---\end{array}$ & $\begin{array}{l}131.30 \\
--- \\
131.26 \\
---\end{array}$ & $\begin{array}{l}130.45 \\
--- \\
130.40 \\
---\end{array}$ \\
\hline $\begin{array}{r}\text { Gly }^{4} S \\
R\end{array}$ & $\begin{array}{l}8.291 \\
8.306\end{array}$ & $\begin{array}{l}3.707 \\
3.897 \\
3.741 \\
3.866\end{array}$ & $\begin{array}{l}-- \\
---\end{array}$ & --- & --- & --- & $\begin{array}{l}--- \\
---\end{array}$ & $\begin{array}{l}45.24 \\
45.33\end{array}$ & --- & --- & --- & --- & --- \\
\hline $\begin{array}{r}\operatorname{Tyr}^{5} S \\
R\end{array}$ & $\begin{array}{l}7.806 \\
7.890\end{array}$ & $\begin{array}{l}4.844 \\
\text { n.a. }\end{array}$ & $\begin{array}{l}2.891 \\
3.055 \\
2.854 \\
3.089\end{array}$ & --- & $\begin{array}{l}7.128 \\
7.147\end{array}$ & $\begin{array}{l}6.820 \\
6.812\end{array}$ & $\begin{array}{l}--- \\
---\end{array}$ & $\begin{array}{l}\text { n.a. } \\
\text { n.a. }\end{array}$ & $\begin{array}{l}\text { n.a. } \\
38.28\end{array}$ & --- & $\begin{array}{l}133.44 \\
133.32\end{array}$ & $\begin{array}{l}118.25 \\
118.38\end{array}$ & --- \\
\hline $\begin{array}{r}\operatorname{Pro}^{6} S \\
R\end{array}$ & --- & $\begin{array}{l}4.426 \\
4.447\end{array}$ & $\begin{array}{l}1.952 \\
2.259 \\
1.959 \\
2.255\end{array}$ & $\begin{array}{l}1.983 \\
1.977\end{array}$ & $\begin{array}{l}3.547 \\
3.755 \\
3.543 \\
3.742\end{array}$ & --- & --- & $\begin{array}{l}\text { n.a.t; } \\
62.81 \mathrm{c} \\
\text { n.a. }\end{array}$ & $\begin{array}{l}32.03 \mathrm{t} ; \\
34.20 \mathrm{c} \\
32.02 \mathrm{t} \\
33.98 \mathrm{c}\end{array}$ & $\begin{array}{l}27.27 \mathrm{t} \\
24.67 \mathrm{c} \\
27.32 \mathrm{t} \\
24.54 \mathrm{c}\end{array}$ & $\begin{array}{l}50.63 \\
50.57\end{array}$ & --- & --- \\
\hline $\begin{array}{r}\operatorname{Ser}^{7} S \\
R\end{array}$ & $\begin{array}{l}8.243 \\
8.252\end{array}$ & $\begin{array}{l}4.338 \\
4.343\end{array}$ & $\begin{array}{l}3.808 \\
3.838 \\
3.821 \\
3.845\end{array}$ & --- & --- & --- & $\begin{array}{l}--- \\
---\end{array}$ & $\begin{array}{l}58.35 \\
\text { n.a. }\end{array}$ & $\begin{array}{l}64.00 \\
63.93\end{array}$ & --- & --- & --- & --- \\
\hline $\begin{array}{r}\mathrm{NH}_{2} S \\
R\end{array}$ & $\begin{array}{l}7.137 \\
7.529 \\
7.131 \\
7.520\end{array}$ & --- & --- & --- & --- & $\begin{array}{l}--- \\
--\end{array}$ & $\begin{array}{l}--- \\
---\end{array}$ & & & & & & \\
\hline
\end{tabular}

Nearly complete assignment of ${ }^{1} \mathrm{H}$ and proton-bearing ${ }^{13} \mathrm{C}$ nuclei in the trans form of dermorphin analogues was obtained from TOCSY, ROESY and ${ }^{1} \mathrm{H} /{ }^{13} \mathrm{C}$ HSQC spectra. NMR-based structural studies of Tyr- $(R)$-Ala- $(S$ and $R)$ - $\alpha$-benzyl- $\beta$-azidoAla-Phe-Asp-Val-Val-Gly- $\mathrm{NH}_{2}$ will be continued.

The presented results demonstrate that $(R)$ - $\alpha$-benzyl- $\beta$-azido(1-piperidinyl)alanine can be used as a successful tool for modulating the affinity and selectivity of peptide ligands. 


\section{Acknowledgments}

This research was supported by the National Science Centre (NCN, Poland), grant Preludium 2 (2011/03/N/ST5/04701).

\section{References}

1. Erspamer, V. Int. J. Dev. Neurosci. 10, 3-30 (1992), http://dx.doi.org/10.1016/0736-5748(92)90003-I

2. Olma, A., et al. Amino Acids 42, 2525-2528 (2012), http://dx.doi.org/10.1007/s00726-011-1055-3:

Kudaj, A., Olma, A. Tetrahedron Lett. 48, 6794-6797 (2007), http://dx.doi.org/10.1016/j.tetlet.2007.07.078

3. Lazarus, L., et al. J. Biol. Chem. 264, 354-362 (1989).

4. Lasota, A., et al. J. Pept. Sci. 20(S1), 171 Abstract P096 (2014). 